\title{
Synthesis of Monodispersed Gold Nanoparticles with Exceptional Colloidal Stability with Grafted Polyethylene Glycol-g-polyvinyl Alcohol
}

\author{
Alaaldin M. Alkilany, ${ }^{1}$ Alaa I. Bani Yaseen, ${ }^{1}$ and Mohammed H. Kailani ${ }^{2}$ \\ ${ }^{1}$ Department of Pharmaceutics \& Pharmaceutical Technology, Faculty of Pharmacy, The University of Jordan, Amman 11942, Jordan \\ ${ }^{2}$ Department of Chemistry, Faculty of Science, The University of Jordan, Amman 11942, Jordan
}

Correspondence should be addressed to Alaaldin M. Alkilany; a.alkilany@ju.edu.jo

Received 23 December 2014; Accepted 3 February 2015

Academic Editor: Subrata Kundu

Copyright ( 2015 Alaaldin M. Alkilany et al. This is an open access article distributed under the Creative Commons Attribution License, which permits unrestricted use, distribution, and reproduction in any medium, provided the original work is properly cited.

\begin{abstract}
Herein, we report the synthesis of spherical gold nanoparticles with tunable core size $(23-79 \mathrm{~nm})$ in the presence of polyethylene glycol-g-polyvinyl alcohol (PEG- $g$-PVA) grafted copolymer as a reducing, capping, and stabilizing agent in a one-step protocol. The resulted PEG- $g$-PVA-capped gold nanoparticles are monodispersed with an exceptional colloidal stability against salt addition, repeated centrifugation, and extensive dialysis. The effect of various synthesis parameters and the kinetic/mechanism of the nanoparticle formation are discussed.
\end{abstract}

\section{Introduction}

Due to their unique optical properties, gold nanoparticles (GNPs) enable promising applications such as imaging, sensing, photothermal ablation of cancer, and drug delivery [1-3]. For various applications, the precise control of particle shape, size, and colloidal stability is essential $[4,5]$. For spherical GNPs, synthetic protocols are available to prepare GNPs with acceptable monodispersity and colloidal stability [2]. Typically, gold salt is reduced by a reducing agent, which may act as capping agent simultaneously. For example, the well-known Frens method employs the reduction of gold salt $\left(\mathrm{HAuCl}_{4}\right)$ in aqueous media by citrate ions at boiling temperature. Citrate ions reduce gold and act as capping agents (physical adsorption) to stabilize the resulting gold colloid $(12-50 \mathrm{~nm})$ by electrical repulsion forces [6]. Stronger reducing agent such as sodium borohydride is used to prepare smaller nanoparticles $(2-10 \mathrm{~nm})$ with physically adsorbed boron species on the surface of GNPs [7]. Both methods result in GNPs that are capped with weakly adsorbed ions (physisorbed) and thus GNPs tend to aggregate upon stresses such as repeated centrifugation, dilution, dialysis, and upon contact with biological media [2]. Postsynthesis surface modification with small stabilizing molecules (e.g., thiolated molecules, amino acids, or proteins) or polymers (e.g., polyvinyl alcohol, polyethylene glycol, and polyelectrolyte) is essential to avoid aggregation and improves colloidal stability [8]. With this in mind, preparing highly stable and tunable GNPs in a one-step synthesis without the need for postsynthesis modification is a clear need. In this regard, special effort in the literature focused on the synthesis of GNPs in the presence of various water-soluble polymers that act as reducing and stabilizing agents simultaneously [911]. Poly(N-vinyl pyrrolidone) (PVP), poly(ethylene glycol) (PEG), and chitosan are examples of polymers used to reduce gold ions and simultaneously stabilize resulting GNPs [9, $11,12]$. Herein, we report the synthesis of monodispersed GNPs using grafted copolymer, namely, polyethylene glycol$g$-polyvinyl alcohol (PEG-g-PVA), which is commercially available, highly water soluble, and biocompatible [13]. The use of grafted copolymers is increasing in the research and industrial practice due to the hybrid functionality of the polymer [14]. For example, PEG-g-PVA was developed by pharmaceutical companies to substitute PVA, which require 
heating and longer time to dissolve and result in increased solution viscosity that is undesired in general processing and special applications. PEG- $g$-PVA is commercially produced in large scale and used in preparing instant-release coatings for pharmaceutical tablets and granules. In this work, we employ PEG-g-PVA as a reducing, capping, and stabilizing agent to prepare GNPs in a one-step protocol. Moreover, PEG-g-PVA allows the synthesis of various sizes of spherical GNPs with excellent monodispersity and exceptional colloidal stability against various stresses such as salt addition, repeated centrifugation, and dialysis.

\section{Experimental Section}

2.1. Materials. Chloroauric acid $\left(\mathrm{HAuCl}_{4} \cdot 3 \mathrm{H}_{2} \mathrm{O}, 99.9 \%\right)$, trisodium citrate, and polyvinyl alcohol (MW 31,000-50,000, 98-99\% hydrolyzed) were obtained from Sigma-Aldrich and used as received. PEG- $g$-PVA (Kollicoat IR 75\%, 25\% polyethylene glycol, and 75\% polyvinyl alcohol, MW $45000 \mathrm{Da})$ and PVA (MW $45000 \mathrm{Da}$ ) were a gift from BASF. All solutions were prepared with purified water $(18.2 \mathrm{M} \Omega \cdot \mathrm{cm})$. Glassware was cleaned with aqua regia and rinsed thoroughly purified water $(18.2 \mathrm{M} \Omega \cdot \mathrm{cm})$. Dialysis cassettes of $\mathrm{MWCO}=$ 100,000 Da were purchased from Spectra Laboratories (Milpitas, CA).

2.2. Instrumentation. Absorption spectra were taken using a UV/VIS spectrophotometer (Spectrascan 80D, Biotech Eng., UK). Transmission electron microscopy (TEM) images were obtained with a Morgagni (Philips, Netherlands) 268 FEI electron microscope operating at $40 \mathrm{kV}$ attached to MegaViewG2 Olympus Soft Imaging Solutions. TEM grids were prepared by drop-casting $10 \mu \mathrm{L}$ of the purified gold nanoparticle dispersions on Formvar coated copper TEM grids (300 mesh, Ted Pella Inc., Redding, CA) and allowing them to dry in air. FTIR spectra were recorded using a Thermo Nicolet NEXUS-670 spectrometer (Nicolet NEXUS-670, Thermo Scientific, Waltham, MA, USA). A micro centrifuge (Eppendorf 5418, Hamburg, Germany) and a LabQuake shaker (Barnstead-Thermolyne, Dubuque, IA) were used in gold nanoparticle synthesis and purification as detailed below.

2.3. Purification of PEG-g-PVA (Kollicoat IR). Purification was conducted by weighing around $5 \mathrm{~g}$ of Kollicoat IR and washing thrice with dichloromethane $(100 \mathrm{~mL}$ at each wash), filtered and left to dry in the oven at $40^{\circ} \mathrm{C}$ for an hour. Afterwards the dried amount was dissolved in $40 \mathrm{~mL}$ DIW until a solution is obtained, which was dialyzed using a cellulose membrane dialysis tube (cutoff MW $=10000$ ) at room temperature overnight against $4.0 \mathrm{~L}$ of DI water. The dialyzed solution was then frozen in liquid nitrogen and lyophilized over two nights to obtain a white powder.

\subsection{Preparation of Citrate-Capped Gold Nanoparticles (Cit-} GNPs). Citrate-capped gold nanoparticles were synthesized using Frens method [6]. An aqueous solution of $0.25 \mathrm{mM} \mathrm{HAuCl}_{4}, 100 \mathrm{~mL}$, was heated in a conical flask and brought to boiling. To the boiling solution, $3.0 \mathrm{~mL}$ of an aqueous solution of $1 \%(\mathrm{w} / \mathrm{w})$ sodium citrate was added.
The heating was maintained until a deep ruby red color appeared (10 minutes), indicating the formation of gold nanoparticles. Transmission electron microscopy UV-Vis spectrophotometry measurements were used to evaluate the prepared nanoparticles.

2.5. Preparation of PEG-g-PVA-Capped Gold Nanoparticles (PEG-g-PVA-GNPs). Typically, PEG- $g$-PVA-GNPs were prepared by boiling $100 \mathrm{~mL}$ of aqueous solution of $1 \% \mathrm{w} / \mathrm{v}$ of PEG- $g$-PVA (Kollicoat IR) and subsequently $0.25 \mathrm{~mL}$ of $0.1 \mathrm{M} \mathrm{HAuCl}_{4}$ was added to the boiling solution. The heating was maintained until a red color appeared (20 minutes), indicating the formation of gold nanoparticles. Transmission electron microscopy and UV-Vis spectrophotometry measurements were used to evaluate the prepared nanoparticles. The same procedure was employed with various concentration of gold salt or polymer at different preparation temperature or $\mathrm{pH}$ to evaluate the effect of these parameters as indicated in the main text.

2.6. Reaction Kinetics Studies. To study the reaction kinetics a typical batch was prepared using $1 \% \mathrm{w} / \mathrm{v}$ PEG- $g$-PVA (Kollicoat IR) solution. $100 \mathrm{~mL}$ of fresh DIW was allowed to heat and $1.00 \mathrm{~g}$ of Kollicoat IR was added; the solution was allowed to boil then $250 \mu \mathrm{L}$ of $\mathrm{HAuCl}_{4}(0.1 \mathrm{M})$ was added at once. The solution was kept boiling for further 15 minutes for complete growth of nanoparticles. Samples were obtained throughout the whole course of the reaction and photograph pictures and UV-Vis spectra were obtained immediately for each sample.

2.7. Preparation of PVA-Capped Gold Nanoparticles (PVAGNPs). Typically, PVA-GNPs were prepared by boiling $100 \mathrm{~mL}$ of aqueous solution of $1 \% \mathrm{w} / \mathrm{v}$ of PVA and subsequently $0.25 \mathrm{~mL}$ of $0.1 \mathrm{M} \mathrm{HAuCl}_{4}$ was added to the boiling solution. The heating was maintained until a red color appeared (20 minutes), indicating the formation of gold nanoparticles. Transmission electron microscopy and UV-Vis spectrophotometry measurements were used to evaluate the prepared nanoparticles.

2.8. Stability against Repeated Centrifugation. The purified gold nanoparticle solutions $(5.0 \mathrm{~mL}$ ) (Cit-GNPs, PVA-GNPs, and PEG- $g$-PVA-GNPs) were centrifuged at $10000 \mathrm{rpm}$ for $10 \mathrm{~min}$, and the resulting pellets were resuspended with DI water. The centrifugation was repeated four times, and then optical photographs and UV-Vis spectra were obtained for all samples after each centrifugation to monitor gold nanoparticle aggregation.

2.9. Stability against Dialysis. A $1.0 \mathrm{~mL}$ purified aliquot of both (Cit-GNPs, PVA-GNPs, and PEG- $g$-PVA-GNPs) solutions was placed in a cellulose membrane dialysis cassette (cutoff $\mathrm{MW}=100000$ ) at room temperature overnight against $4.0 \mathrm{~L}$ of DI water. After dialysis, optical photographs and UV-Vis spectra were obtained for solutions in the cassettes to monitor gold nanoparticles stability against aggregation. 


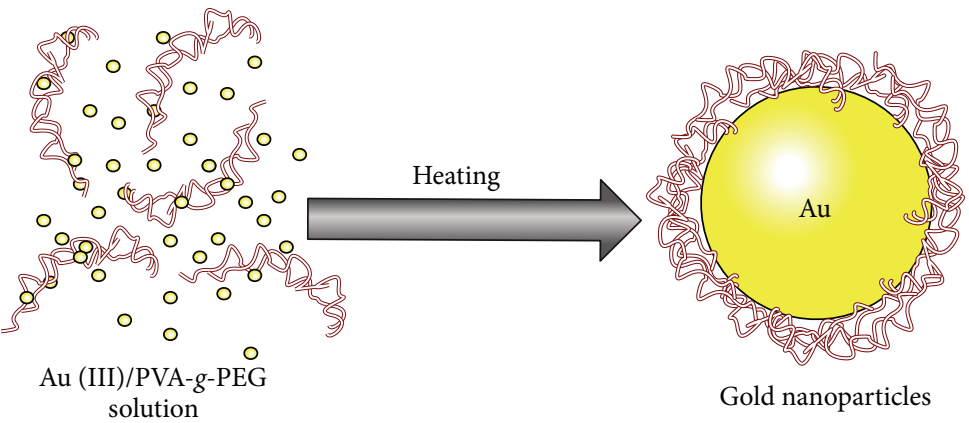

(a)

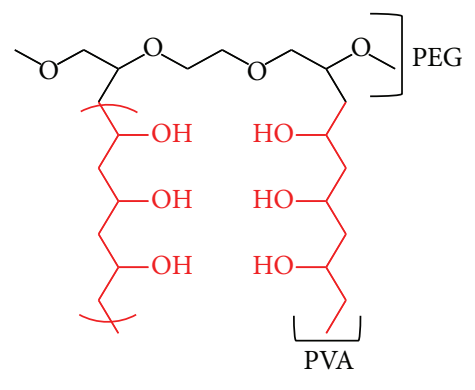

PVA- $g$-PEG

(b)

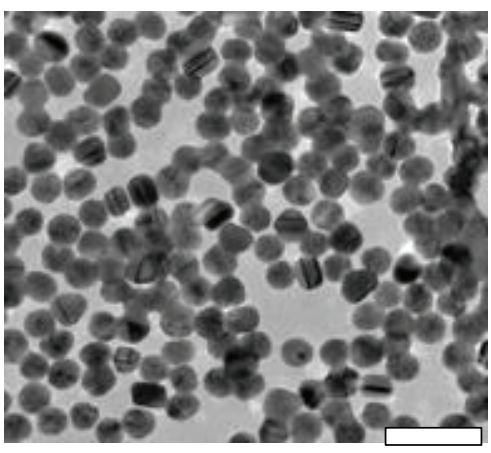

(c)

FIGURE 1: (a) Schematic of the one-step synthesis of gold nanoparticles from gold salt and PEG- $g$-PVA polymer as a reducing, capping, and stabilizing agent. (b) Molecular structure of the used PEG- $g$-PVA graft copolymer. (c) Transmission electron microscope image of PEG- $g$ PVA-capped GNPs (Scale bar $=100 \mathrm{~nm})$.

2.10. Stability against Salt Addition. The purified gold nanoparticle solutions $(1.0 \mathrm{~mL})$, both (Cit-GNPs, PVA-GNPs, and PEG- $g$-PVA-GNPs), were centrifuged at $10000 \mathrm{rpm}$ for $10 \mathrm{~min}$, and the resulting pellets were resuspended with solution of $\mathrm{NaCl}$ with different concentrations $(1,0.5,0.1,0.05$, and $0.01 \mathrm{M}$ ). Sonication was applied for 1 minutes and then photograph pictures and UV-Vis spectra were obtained immediately.

\section{Results and Discussion}

The aim of this work is to evaluate to feasibility of using polyethylene glycol- $g$-polyvinyl alcohol (PEG- $g$-PVA) grafted copolymer as a reducing, capping, and stabilizing agent in a one-step synthesis of GNPs. The rationale of using PEG- $g$-PVA is based on the unique structure of this grafted polymer, in which the PVA brushes (which may act as reducing and capping moieties) are grafted from PEG backbone (which may act as a reducing and strong stabilizing agent). Molecular structure of PEG- $g$-PVA is shown in Figure 1. Boiling an aqueous solution of gold salt $\left(\mathrm{HAuCl}_{4}\right)$ in presence of PEG- $g$-PVA resulted in the formation of deep-red solution indicating the formation of GNPs (particle diameter $=23.2 \pm 1.1 \mathrm{~nm})$. The resulting GNPs were monodispersed (polydispersity index $<5 \%$ ) as evident from TEM analysis (Figure 1(c)). Since our protocol employs the use of PEG-g-PVA in absence of any other common reducing agents such as hydrides or citrate, we attribute the reduction of gold salt to the presence of the polymer itself as a reducing agent. To exclude an artifact from impurities in the commercially used PEG- $g$-PVA in our synthesis, the polymer was purified extensively by dialysis followed by lyophilization as detailed in the experimental section. Both "as is" and "purified" PEG$g$-PVA resulted in similar GNPs (data not shown), indicating that the PEG- $g$-PVA polymer itself initiates the reduction of gold ions and not other associated impurities. The ability of PEG- $g$-PVA to reduce gold ions is not surprising since pure PEG or PVA can act as a reducing agent for metal ions [15]. Longenberger and Mills reported that metal salts of $\mathrm{Au}, \mathrm{Pd}$, and $\mathrm{Ag}$ are transformed into metal nanoparticles in aqueous solution containing either PEG or PVA polymer at room temperature [15]. However, resulting metallic nanoparticles in their work were formed in (8-144) hours with a broad particle size distribution (in our case, the reaction is much faster $\sim 30$ minutes at boiling condition and results in a GNPs with excellent monodispersity as evident from the TEM image in Figure 1(c)).

Since the reduction of gold ions should be coupled with the oxidation of the PEG- $g$-PVA, we attempted to follow any possible oxidation of the polymer using FTIR analysis. Figure 2 shows the FTIR spectra of purified PEG$g$-PVA in comparison with thoroughly purified PEG- $g$-PVAcapped gold nanoparticles (PEG-g-PVA-GNPs). The characteristic vibration bands of $\mathrm{PEG}-\mathrm{g}$-PVA can be assigned to the vibration of $\mathrm{C}-\mathrm{OH}, \mathrm{C}-\mathrm{H}, \mathrm{C}=\mathrm{O}$, and $\mathrm{C}-\mathrm{O}$ bonds as labeled in Figure 2. Above $3000 \mathrm{~cm}^{-1}$, a broad and very 


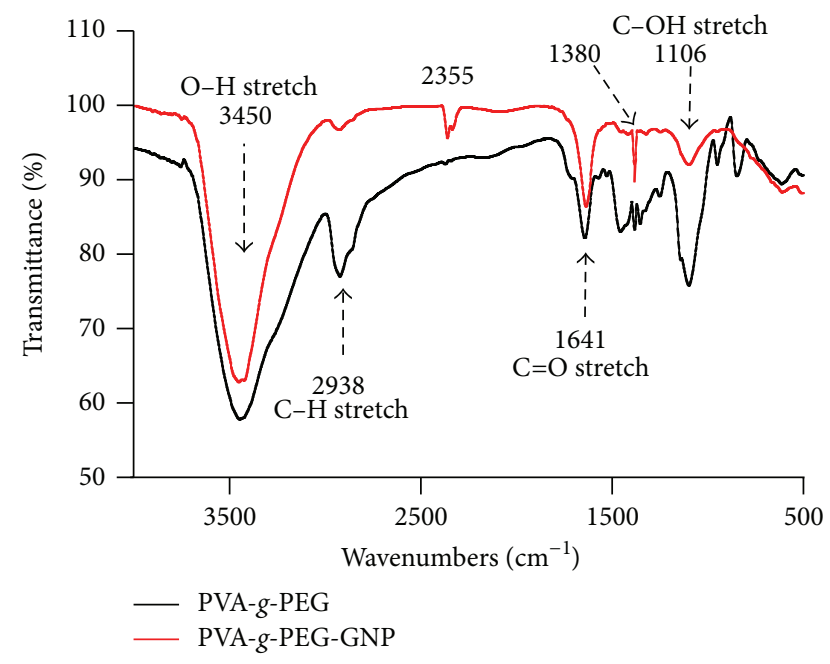

FIGURE 2: FTIR spectra of purified graft copolymer (PEG-g-PVA) versus purified gold nanoparticles (PEG- $g$-PVA-GNPs).

strong absorption band, which derives from the stretching vibration of the hydroxyl group (at $3650-2800 \mathrm{~cm}^{-1}$ ), is clear in both spectra. In the wavenumber range $3000-2800 \mathrm{~cm}^{-1}$ PEG- $g$-PVA exhibits absorbance bands at $2938 \mathrm{~cm}^{-1}$ that corresponds to the stretching vibrations of $-\mathrm{CH}_{2}$ groups. Characteristic vibration bands of carbonyl function (originally in the PVA moieties due to incomplete hydrolysis) are found at $1641 \mathrm{~cm}^{-1}$. The bending vibrations of the $\mathrm{C}-\mathrm{H}$ bands are located at $1380 \mathrm{~cm}^{-1}$. The two bands found at $1106 \mathrm{~cm}^{-1}$ are assigned to the $\mathrm{C}-\mathrm{O}$ stretching. Unfortunately, we were not able to detect significant changes in both spectra for the polymer and the resulting nanoparticles. We did expect to see a vibration peaks related to conjugated polyenes and/or nonconjugated polyenes as a result of PVA segment oxidative degradation or vibration peaks associated with the formation of acid and/or aldehydes as a result of PEG oxidation and chain scission $[15,16]$. The low sensitivity of our FTIR analysis to detect significant changes in both spectra could be explained by the low number of functional groups in the polymer that are involved in the redox chemistry (polymeric functional groups are in excess compared to gold ions), which hindered unveiling the molecular mechanism of the redox chemistry that involved in the reduction of gold ions by PEG$g$-PVA.

Interestingly, the particle size and thus the optical properties of PEG- $g$-PVA-capped GNPs can be tuned readily by varying the (polymer: Au) ratio. GNPs with various particle size $(d=23.2 \pm 1.1-79.3 \pm 9.0 \mathrm{~nm})$ were prepared by varying the polymer content from $0.125 \%$ to $2.0 \% \mathrm{w} / \mathrm{w}$ at a constant gold salt level $(1.0 \mathrm{mM})$ as evident from various solution color (red-purple-blue) and a clear red-shift in corresponding plasmon peaks in their UV-Vis $\left(\lambda_{\max }\right.$ red-shifts in the range of $529-580 \mathrm{~nm}$ ) without significant broadening (Figure 3). Moreover, the resulting spherical GNPs showed excellent monodispersity as evident from TEM analysis (Figure 3). It is worth mentioning that using polymer content less than $0.125 \% \mathrm{w} / \mathrm{w}$ resulted in the formation of polydispersed and large nanoparticles which aggregate readily, where the use of higher polymer concentrations $(>3 \% \mathrm{w} / \mathrm{w})$ resulted in smaller nanoparticles which is difficult to purify by centrifugation due to the higher viscosity of solution at higher polymer levels (data not shown). The ability to control gold nanoparticle's size and optical properties via polymer content was demonstrated for related systems where poly(N-vinyl-2pyrrolidone) was used to prepare GNPs in one-step synthesis [12].

Beside the effect of (polymer: $\mathrm{Au}$ ) ratio, optimization studies were performed to evaluate the effect of temperature and $\mathrm{pH}$ on the resulting PEG- $g$-PVA-capped GNPs in term of polydispersity as judged by the broadness of their UVVis spectra. We found that the boiling condition $\left(100^{\circ} \mathrm{C}\right)$ is required to insure a sharp plasmon peak around $530 \mathrm{~nm}$, where decreasing the reaction temperature results in broader Plasmon peak as evident from Figure 4(a). Higher temperature is well-known to result in faster and controlled reduction of $\mathrm{Au}(\mathrm{III})$ ions and thus more homogenous nucleation and monodispersed GNPs [17]. Neutral pH (around 6.5) was found optimal to prepare monodispersed GNPs with the narrowest plasmon peak (Figure 4(b)) where either basic and acidic conditions increase the polydispersity of GNPs as evident from broader plasmon peak at $\mathrm{pH}=1$ or $\mathrm{pH}=$ 9. Interestingly, at $\mathrm{pH}=1.2$ (highly acidic), no nanoparticles were formed as evident from the absence of the plasmon peak in the UV-Vis spectra and the clear solution in Figure 4(b). Our finding disagree with the case of reduction of gold salt by ascorbic acid in aqueous medium where low $\mathrm{pH}$ results in well-dispersed and uniform GNPs while higher $\mathrm{pH}$ results in larger GNPs and ensembles of fine Au colloid [18]. However, both systems (reduction by the PEG- $g$-PVA or by ascorbic acid) are completely different for direct comparison. One explanation of the dependency of GNPs properties on solution $\mathrm{pH}$ is the fact that reaction $\mathrm{pH}$ could affect the redox chemistry of the secondary hydroxyl groups in PVA segment and/or the ether groups in the PEG segment. Other explanations could be that the complexation of the hydroxyl or ether groups in the PEG- $g$-PVA with gold metal is altered by the solution $\mathrm{pH}$. The later explanation is supported by the reported speciation of gold ions as function of solution $\mathrm{pH}$ as aqueous $\mathrm{HAuCl}_{4}$ consists of $\left[\mathrm{AuCl}_{x}(\mathrm{OH})_{4-x}\right]^{-}$, where $(x<2)$ at low $\mathrm{pH}$ and $(x \geq 2)$ at high $\mathrm{pH}[18]$. However, the exact mechanism of molecular interaction between the polymer and the gold ion prior to reduction and the consequent reduction to form GNPs is not elusive at the time being and should be the subject of future research.

At optimum conditions $\left(\mathrm{pH}=6.8, T=100^{\circ} \mathrm{C}, 1 \% \mathrm{w} / \mathrm{w}\right.$ polymer), the kinetics of the formation of PEG- $g$-PVA-GNPs was studied by following the development of the plasmon peak using UV-Vis spectrophotometer. Figure 5 shows the color and the UV-Vis spectra of the reaction solution at various time points (one-minute intervals). At the first 5 minutes, grayish color showed up with very broad plasmon peak with low intensity around $500-600 \mathrm{~nm}$, indicating the formation of small "nuclei" of noble gold [19]. After this stage, violet solution color was observed and the plasmon peak intensity increased with blue-shifted to lower wavelength as the heating maintained (5-15 minutes). After about 


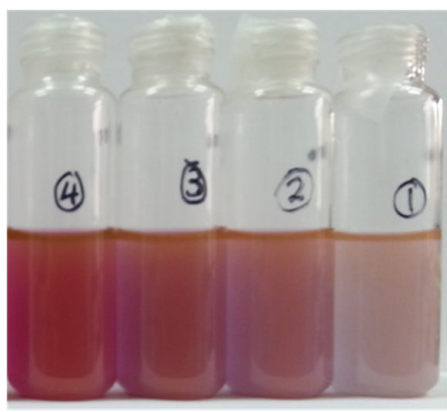

(a)

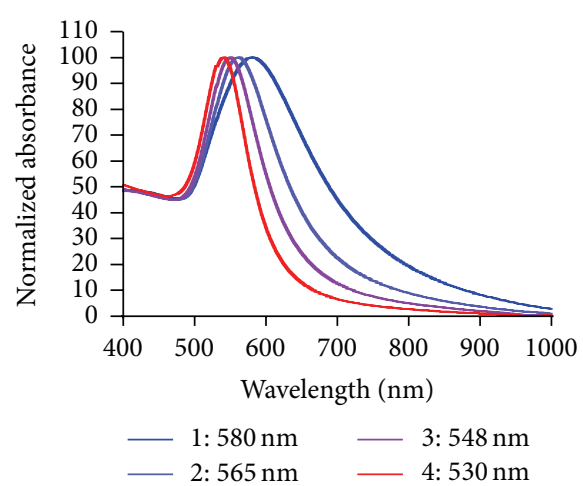

(b)
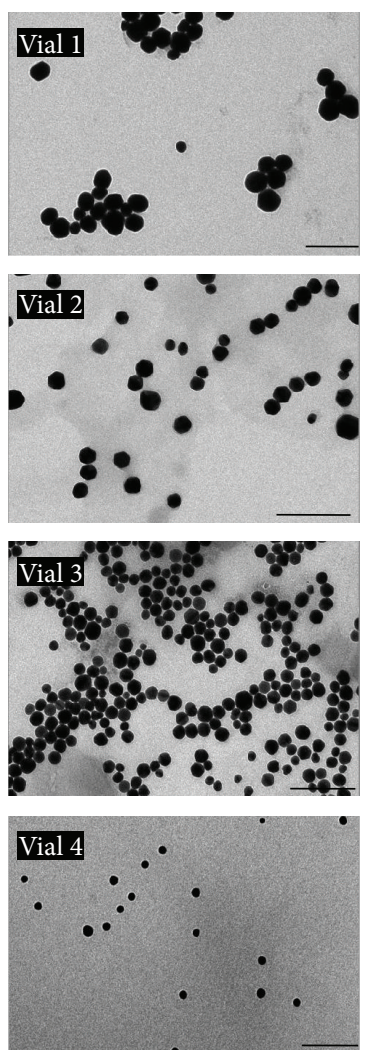

FIgURE 3: Photograph (a), UV-Vis spectra (b), and TEM images (right panel) of PEG-g-PVA-capped GNPs prepared using various (polymer: $\mathrm{Au}$ ) ratio $(0.125,0.25,0.5$, and $1 \mathrm{w} / \mathrm{w} \%$ for vials, spectra or TEM images from 1 to 4 , resp.). Particle size diameters in TEM images are $79.3 \pm 9,62.5 \pm 6.3,44.3 \pm 3.1$, and $23.2 \pm 1.1 \mathrm{~nm}$ for images $1,2,3$, and 4, respectively. Scale bar in all images $=200 \mathrm{~nm}$.
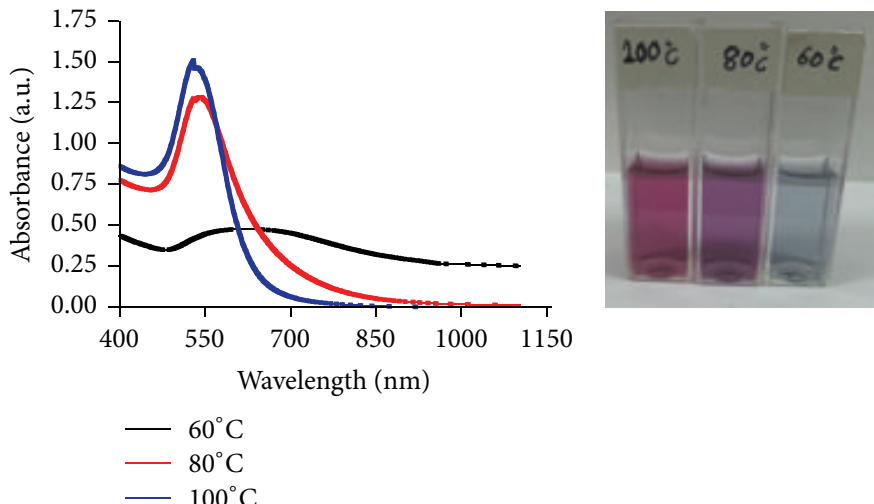

(a)
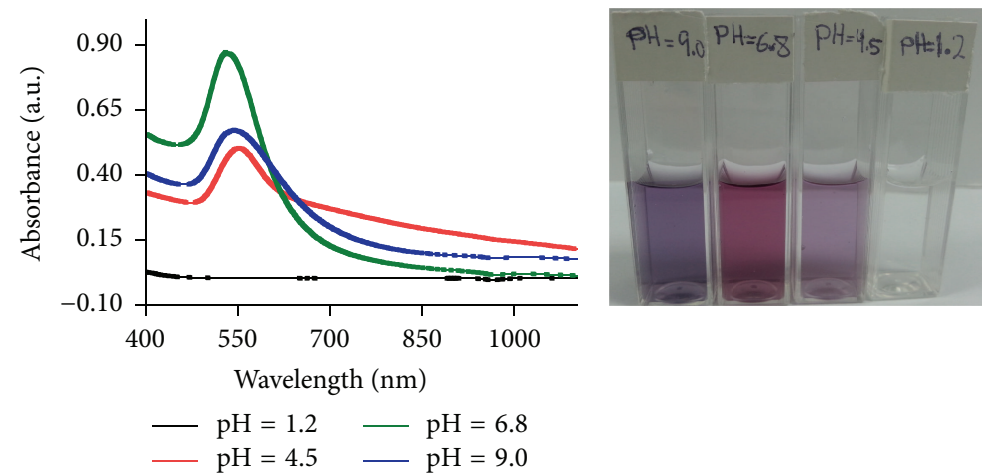

(b)

FIgUre 4: UV-Vis spectra and photographs of PEG- $g$-PVA-GNPs prepared at various temperature (a) and pH values (b) as labeled. 


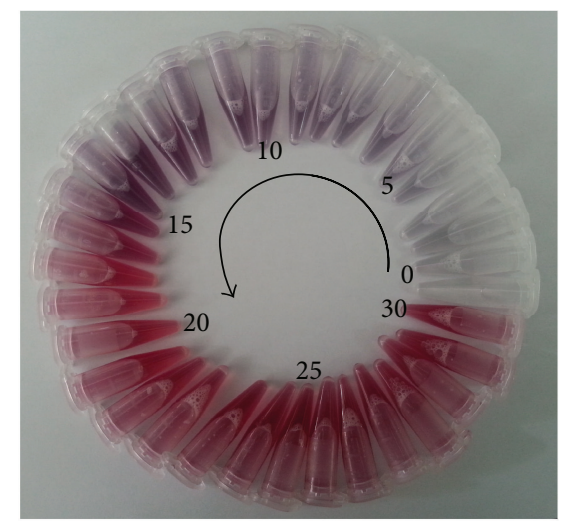

(a)

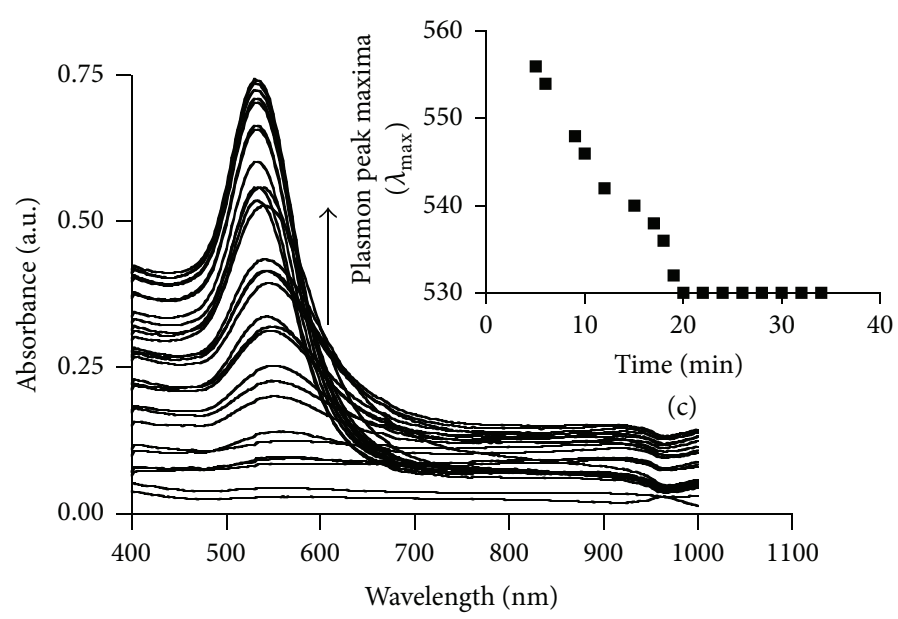

(b)

FIGURE 5: Photographs (a) and UV-Vis spectra (b) of PEG-g-PVA-GNPs at various reaction time intervals. (c) Represents the time-dependent blue-shift of the LSPR band in figure (b).

15 minutes, solution turned to reddish in color as shown in Figure 5(a) and remained visually indistinguishable with constant plasmon peak maxima $\left(\lambda_{\max }\right)$ around $530 \mathrm{~nm}$ after 20 minutes as shown in Figures 5(b) and 5(c). Interestingly, we observed a continuous blue-shift in the localized surface plasmon resonance (LSPR) of GNPs during our reaction. This observation could be the result of continuous decreased in nanoparticle size polydispersity upon continuous consumption of gold nuclei to grow larger monodispersed GNPs via the Ostwald ripening and/or collision coalescence mechanisms [20]. Another explanation is based on the continuous deaggregation of nanoparticle "assembles" upon heating [21] due to enhanced adsorption of stabilizing polymer on the surface of GNPs to eliminate plasmon coupling effect, which is associated with a red-shift in the LSPR with significant broadening $[1,19]$. Sardar and Shumaker-Parry reported the synthesis of GNPs using 9-orabicyclo[3.3.1]nonane where they observed a red-shift followed by a blue-shift in the LSPR peak, which was explained by a decrease in particle size polydispersity and dispersion [22]. An extensive TEM analysis coupled with an in situ particle growth quenching are necessary to unveil the exact explanation for the observed blue-shift and should be the subject of future contribution.
Colloidal stability of nanoparticles is a critical factor dictating its physical, chemical, and biological properties [4]. For various applications, maintaining an excellent colloidal stability is advantageous. Unfortunately, many synthetic routes of preparing gold nanoparticles do not ensure a high stability of the resulting nanoparticles. For example, citrate-capped gold nanoparticles (Cit-GNPs) as prepared using the classical Frens method are meta-stable at best, which usually aggregate upon salt addition, dialysis, or repetitive centrifugation. With this in mind, postsynthesis surface functionalization is required to enhance their colloidal stability. Herein, the resulting PEG- $g$-PVA-GNPs were found to have exceptional colloidal stability "as prepared" without the need for any further surface modification. The colloidal stability of three types of nanoparticles (Cit-GNPs, PVA-GNPs, and PEG- $g$ PVA-GNPs) against salt addition, repeated centrifugation, and dialysis was evaluated. The labile nature of Cit-GNPs against salt addition is clearly shown in Figure 6(a), where nanoparticle aggregation was induced at $\mathrm{NaCl}$ concentration of $0.1 \mathrm{M}$, in agreement with published reports [23]. The aggregation of Cit-GNPs is evident from the color change from red to blue and associated red-shift and broadening in their LSPR peak. PVA-GNPs showed superior stability compared 


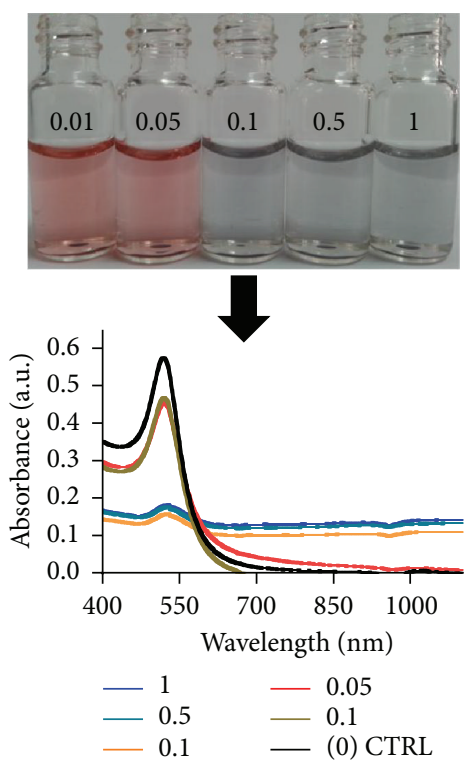

(a)

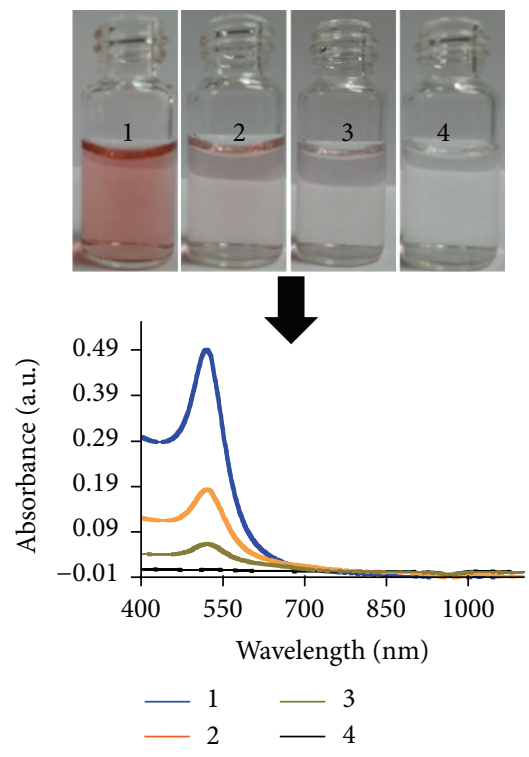

(d)

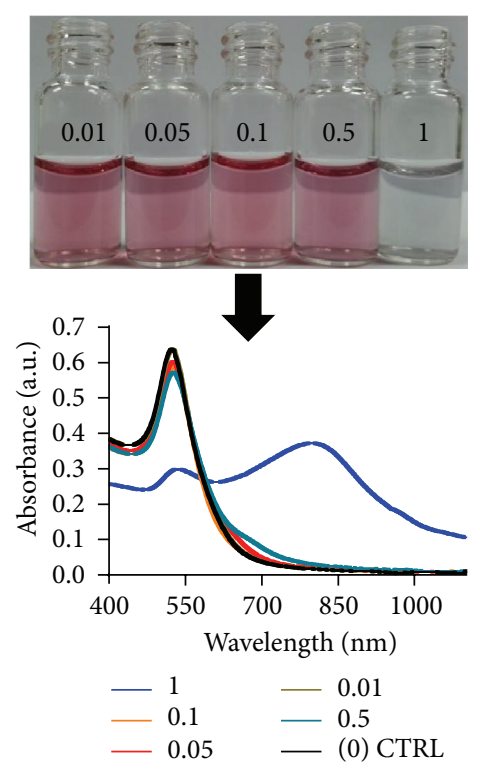

(b)

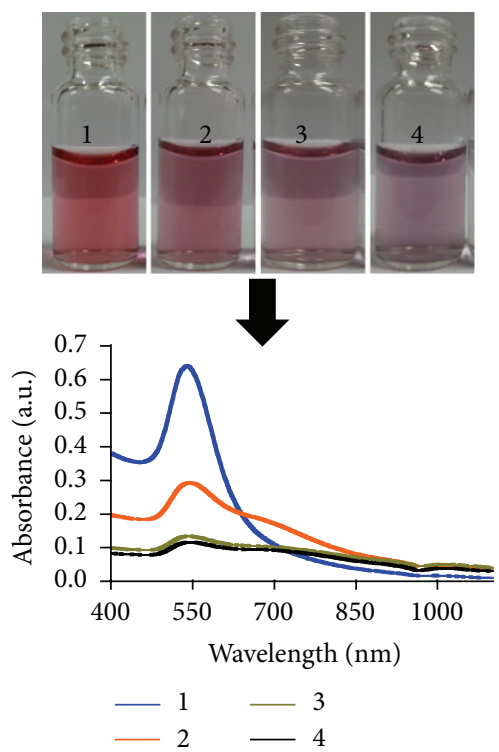

(e)

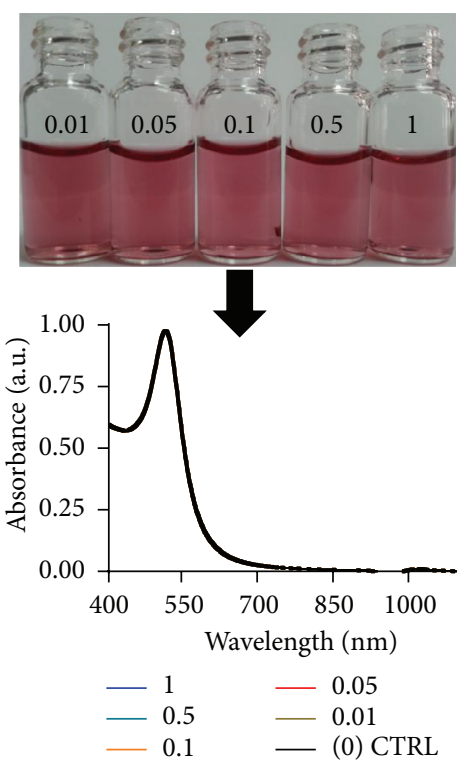

(c)

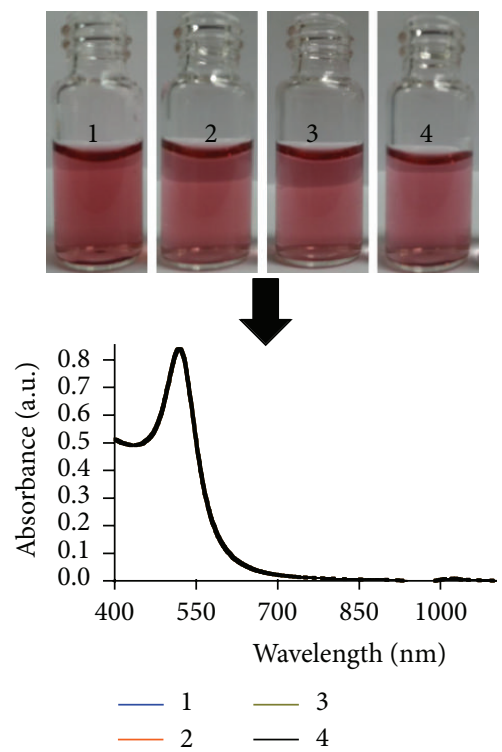

(f)

Figure 6: Colloidal stability evaluation of Cit-GNPs ((a) and (d)), PVA-GNPs ((b) and (e)), and PEG-PVA-GNPs ((c) and (f)) against salt addition (upper panel, (a), (b), and (c)) or against repeated centrifugation (lower panel, (d), (e), and (f)). Photograph and corresponding $\mathrm{UV}-\mathrm{Vis}$ spectra (indicated by arrows) are shown for each case.

to Cit-GNPs (Figure 6(b)), where aggregation starts at much higher concentration (stable at $0.5 \mathrm{NaCl}$ and aggregated at 1.0 M NaCl). Interestingly, PEG- $g$-PVA-GNPs show exceptional colloidal stability against salt addition as evident from the constant red color of their solutions and the superimposing UV-Vis spectra in Figure 6(c). Similar to the excellent colloidal stability against salt addition, PEG- $g$-PVA-GNPs show exceptional stability against repeated centrifugation as shown in Figures 6(d)-6(f). While a significant aggregation is induced upon the second centrifugation; in the case of Cit-GNPs and PVA-GNPs, no aggregation was observed in the case of PEG- $g$-PVA-GNPs as evident from constant red solution color and UV-Vis spectra (Figure 6(f)). Moreover, similar trend was observed when the colloidal stability of the three types of GNPs was evaluated against dialysis (Figure 7). Our colloidal stability evaluation clearly highlights the exceptional stability of the prepared PEG-g-PVA-GNPs, which may be explained by the presence of polymeric shell of both PEG-PVA on the surface of GNPs that act as a capping agent and stabilizing agent considering that both PVA and PEG are well-known surface stabilizers and capping agent for various colloidal systems $[24,25]$. 


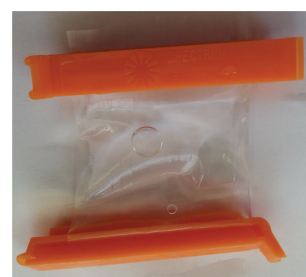

(a)

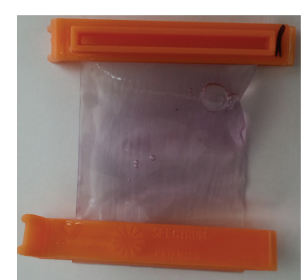

(b)

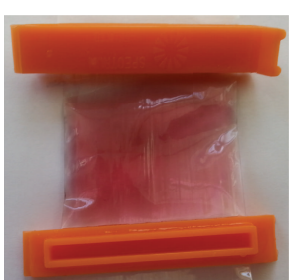

(c)

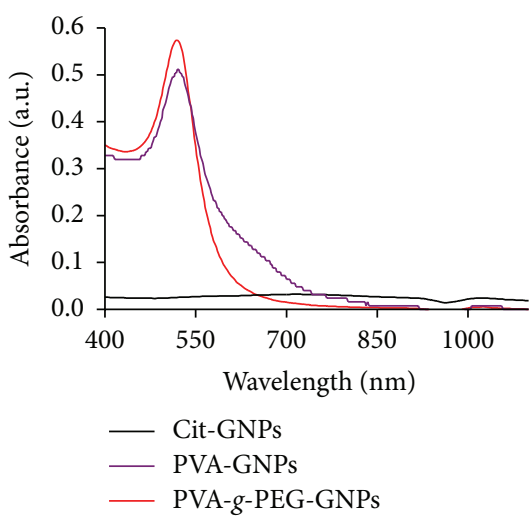

(d)

FIgURE 7: Colloidal stability evaluation of Cit-GNPs (a), PVA-GNPs (b), and PEG-g-PVA-GNPs (c) against dialysis. Photograph of the dialysis cassette showing the color of the resulting nanoparticle solution and the corresponding UV-Vis spectra (as labeled in the graph) are shown.

\section{Conclusion}

In conclusion, polyethylene glycol- $g$-polyvinyl alcohol (PEG$g$-PVA) grafted copolymer was employed successfully as a reducing, capping, and stabilizing agent in a one-step protocol to prepare monodispersed gold nanoparticles with tunable core size $(23-79 \mathrm{~nm})$. The use of PEG- $g$-PVA to prepare gold nanoparticles showed exceptional colloidal stability against various stresses such as salt addition, repeated centrifugation, and dialysis compared to gold colloid prepared with PVA or citrate ions for comparison. The synthetic protocol can be fine-tuned by various parameters such as $\mathrm{pH}$, $\mathrm{Au}$ : polymer ratio, and reaction temperature.

\section{Conflict of Interests}

The authors declare that there is no conflict of interests regarding the publication of this paper.

\section{References}

[1] E. C. Dreaden, A. M. Alkilany, X. Huang, C. J. Murphy, and M. A. El-Sayed, "The golden age: gold nanoparticles for biomedicine," Chemical Society Reviews, vol. 41, no. 7, pp. 27402779, 2012.

[2] M.-C. Daniel and D. Astruc, "Gold nanoparticles: assembly, supramolecular chemistry, quantum-size-related properties, and applications toward biology, catalysis, and nanotechnology," Chemical Reviews, vol. 104, no. 1, pp. 293-346, 2004.

[3] R. Cao-Milán and L. M. Liz-Marzán, "Gold nanoparticle conjugates: recent advances toward clinical applications," Expert Opinion on Drug Delivery, vol. 11, no. 5, pp. 741-752, 2014.
[4] J. W. Krumpfer, T. Schuster, M. Klapper, and K. Müllen, "Make it nano-Keep it nano," Nano Today, vol. 8, no. 4, pp. 417-438, 2013.

[5] J. Zhou, J. Ralston, R. Sedev, and D. A. Beattie, "Functionalized gold nanoparticles: synthesis, structure and colloid stability," Journal of Colloid and Interface Science, vol. 331, no. 2, pp. 251262, 2009.

[6] G. Frens, "Controlled nucleation for the regulation of the particle size in monodisperse gold suspensions," Nature, vol. 241, no. 105, pp. 20-22, 1973.

[7] N. R. Jana, "Gram-scale synthesis of soluble, near-monodisperse gold nanorods and other anisotropic nanoparticles," Small, vol. 1, no. 8-9, pp. 875-882, 2005.

[8] R. A. Sperling and W. J. Parak, "Surface modification, functionalization and bioconjugation of colloidal Inorganic nanoparticles," Philosophical Transactions of the Royal Society A: Mathematical, Physical and Engineering Sciences, vol. 368, no. 1915, pp. 1333-1383, 2010.

[9] S. K. Seol, D. Kim, S. Jung, W. S. Chang, and J. T. Kim, “One-step synthesis of PEG-coated gold nanoparticles by rapid microwave heating," Journal of Nanomaterials, vol. 2013, Article ID 531760, 6 pages, 2013.

[10] R. Stiufiuc, C. Iacovita, R. Nicoara et al., "One-step synthesis of PEGylated gold nanoparticles with tunable surface charge," Journal of Nanomaterials, vol. 2013, Article ID 146031, 7 pages, 2013.

[11] J. Shan and H. Tenhu, "Recent advances in polymer protected gold nanoparticles: synthesis, properties and applications," Chemical Communications, no. 44, pp. 4580-4598, 2007.

[12] C. E. Hoppe, M. Lazzari, I. Pardiñas-Blanco, and M. A. LópezQuintela, "One-step synthesis of gold and silver hydrosols using poly(N-vinyl-2- pyrrolidone) as a reducing agent," Langmuir, vol. 22, no. 16, pp. 7027-7034, 2006. 
[13] F. F. Heuschmid, S. Schneider, P. Schuster, B. Lauer, and B. V. Ravenzwaay, "Developmental toxicity of polyethylene glycol-gpolyvinyl alcohol grafted copolymer in rats and rabbits," Food and Chemical Toxicology, vol. 51, supplement 1, pp. S14-S23, 2013.

[14] C. Feng, Y. Li, D. Yang, J. Hu, X. Zhang, and X. Huang, "Welldefined graft copolymers: from controlled synthesis to multipurpose applications," Chemical Society Reviews, vol. 40, no. 3, pp. 1282-1295, 2011.

[15] L. Longenberger and G. Mills, "Formation of metal particles in aqueous solutions by reactions of metal complexes with polymers," Journal of Physical Chemistry, vol. 99, no. 2, pp. 475-478, 1995.

[16] Z. Peng and L. X. Kong, "A thermal degradation mechanism of polyvinyl alcohol/silica nanocomposites," Polymer Degradation and Stability, vol. 92, no. 6, pp. 1061-1071, 2007.

[17] J. Turkevich, P. C. Stevenson, and J. Hillier, "A study of the nucleation and growth processes in the synthesis of colloidal gold," Discussions of the Faraday Society, vol. 11, pp. 55-75, 1951.

[18] S. Wang, K. Qian, X. Bi, and W. Huang, "Influence of speciation of aqueous $\mathrm{HAuCl}_{4}$ on the synthesis, structure, and property of Au colloids," The Journal of Physical Chemistry C, vol. 113, no. 16, pp. 6505-6510, 2009.

[19] K. L. Kelly, E. Coronado, L. L. Zhao, and G. C. Schatz, “The optical properties of metal nanoparticles: the influence of size, shape, and dielectric environment," Journal of Physical Chemistry B, vol. 107, no. 3, pp. 668-677, 2003.

[20] X. Jia, J. Listak, V. Witherspoon, E. E. Kalu, X. Yang, and M. R. Bockstaller, "Effect of matrix molecular weight on the coarsening mechanism of polymer-grafted gold nanocrystals," Langmuir, vol. 26, no. 14, pp. 12190-12197, 2010.

[21] Y.-C. Wang and S. Gunasekaran, "Spectroscopic and microscopic investigation of gold nanoparticle nucleation and growth mechanisms using gelatin as a stabilizer," Journal of Nanoparticle Research, vol. 14, no. 10, article 1200, 2012.

[22] R. Sardar and J. S. Shumaker-Parry, "Spectroscopic and microscopic investigation of gold nanoparticle formation: ligand and temperature effects on rate and particle size," Journal of the American Chemical Society, vol. 133, no. 21, pp. 8179-8190, 2011.

[23] S. Dominguez-Medina, J. Blankenburg, J. Olson, C. F. Landes, and S. Link, "Adsorption of a protein monolayer via hydrophobic interactions prevents nanoparticle aggregation under harsh environmental conditions," ACS Sustainable Chemistry \& Engineering, vol. 1, no. 7, pp. 833-842, 2013.

[24] A. S. Karakoti, S. Das, S. Thevuthasan, and S. Seal, "PEGylated inorganic nanoparticles," Angewandte Chemie International Edition, vol. 50, no. 9, pp. 1980-1994, 2011.

[25] H. Tyagi, A. Kushwaha, A. Kumar, and M. Aslam, "PVA stabilized gold nanoparticles using ascorbic acid as a reducing agent," in Proceedings of the 55th DAE Solid State Physics Symposium, vol. 1349 of AIP Conference Proceedings, pp. 419-420, Manipal, India, July 2011. 

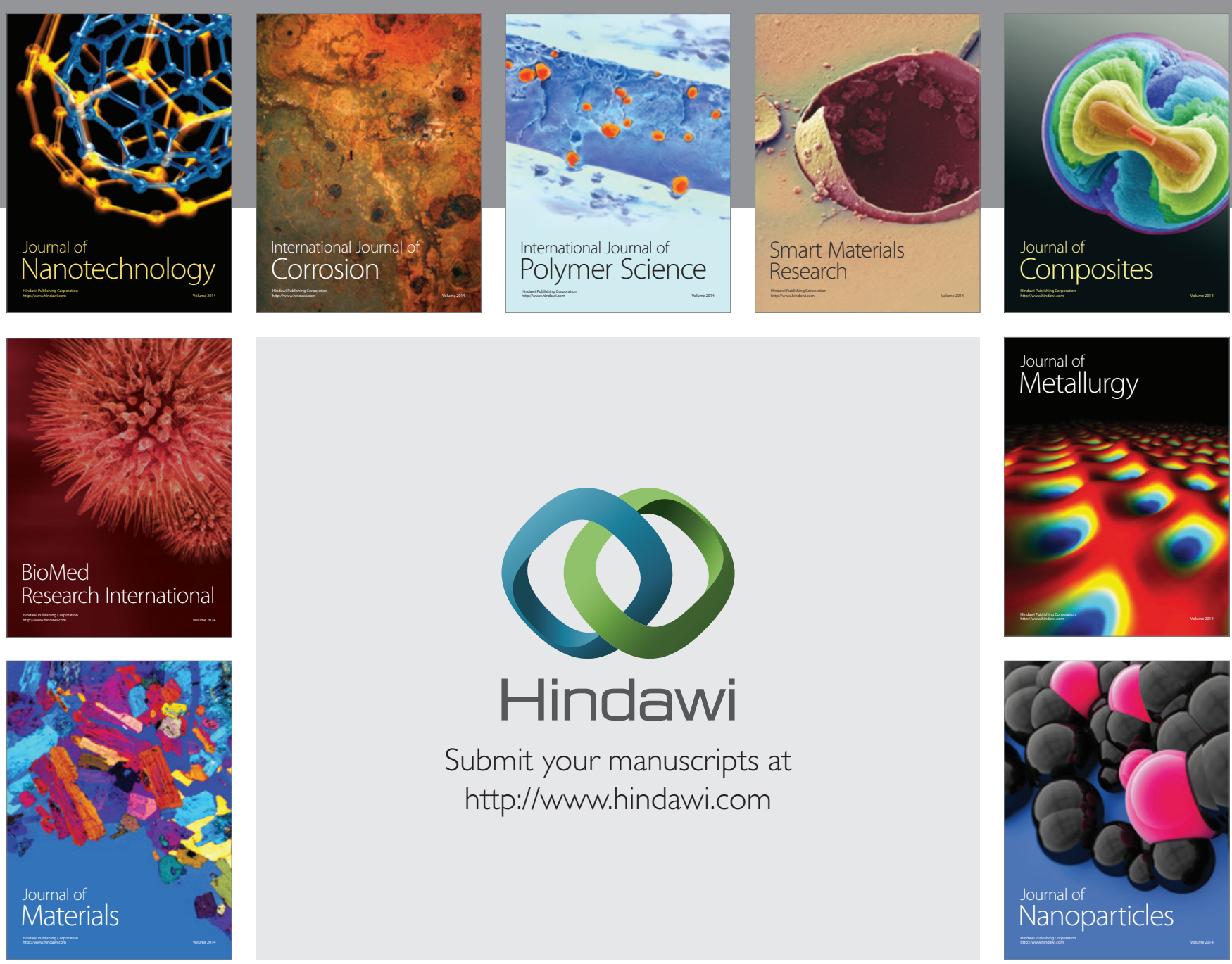

Submit your manuscripts at http://www.hindawi.com
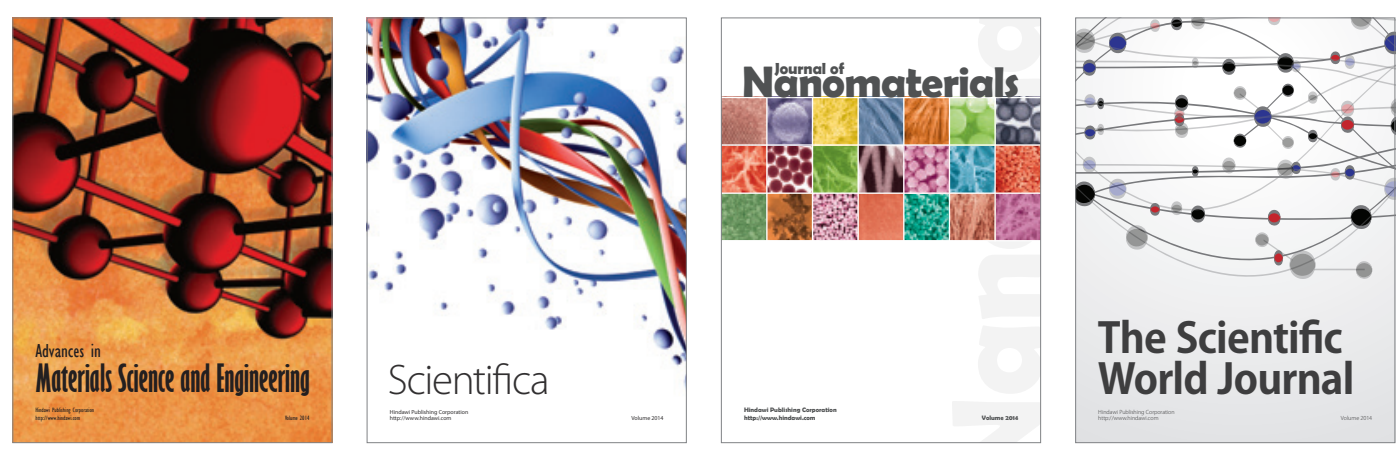

\section{The Scientific World Journal}
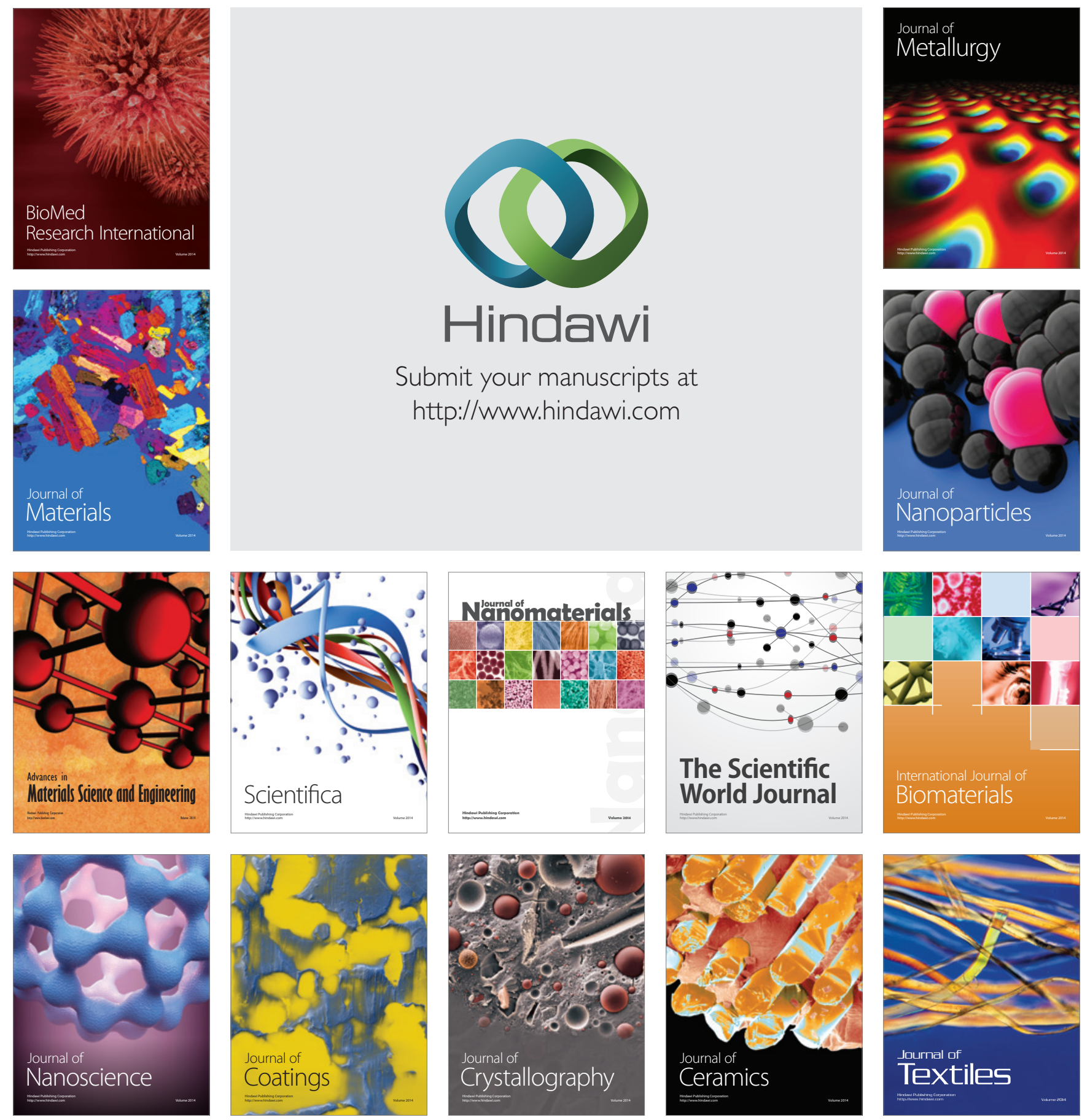